\title{
Random Hamiltonians Ergodic in All But One Direction
}

\author{
H. Englisch ${ }^{1}$, W. Kirsch ${ }^{2}$, M. Schröder ${ }^{1}$, and B. Simon ${ }^{3}$ \\ ${ }^{1}$ NTZ, Karl-Marx-Universität, DDR-7010 Leipzig, German Democratic Republic \\ ${ }^{2}$ Institut für Mathematik and SFB 237, Ruhr-Universität, D-4630 Bochum, \\ Federal Republic of Germany \\ ${ }^{3}$ Department of Mathematics, Physics and Astronomy, California Institute of Technology, \\ Pasadena, CA 91125, USA
}

\begin{abstract}
Let $V_{\omega}^{(1)}$ and $V_{\omega}^{(2)}$ be two ergodic random potentials on $\mathbb{R}^{d}$. We consider the Schrödinger operator $H_{\omega}=H_{0}+V_{\omega}$, with $H_{0}=-\Delta$ and for $x=\left(x_{1}, \ldots, x_{d}\right)$

$$
V_{\omega}(x)=\left\{\begin{array}{lll}
V_{\omega}^{(1)}(x) & \text { if } \quad x_{1}<0 \\
V_{\omega}^{(2)}(x) & \text { if } & x_{1} \geqq 0
\end{array} .\right.
$$

We prove certain ergodic properties of the spectrum for this model, and express the integrated density of states in terms of the density of states of the stationary potentials $V_{\omega}^{(1)}$ and $V_{\omega}^{(2)}$. Finally we prove the existence of the density of surface states for $H_{\omega}$.
\end{abstract}

\section{Introduction}

In this paper we consider Schrödinger operators $H_{\omega}=H_{0}+V_{\omega}$ with random potential $V_{\omega}$ on $L^{2}\left(\mathbb{R}^{d}\right)$. The random potential $V_{\omega}$ we consider has different behavior in the left and right half space. More precisely, there are two ergodic random fields $V_{\omega}^{+}$and $V_{\omega}^{-}$on $\mathbb{R}^{d}$ such that $V_{\omega}$ agrees with $V_{\omega}^{+}$in one half space and with $V_{\omega}^{-}$in the complementary half space. To be specific we assume $V_{\omega}(x)=V_{\omega}^{+}$for $x_{1} \geqq 0$ and $V_{\omega}(x)=V_{\omega}^{-}(x)$ for $x_{1}<0$.

Thus $V_{\omega}$ is not an ergodic potential (unless $V_{\omega}^{ \pm}$happen to agree). Consequently, the general theory of ergodic potentials (see e.g. $[4,2,10]$ and references therein) does not apply. For example, a priori the spectrum $\sigma\left(H_{\omega}\right)$ may depend on $\omega$. In fact, Molcanov and Seidel [15] consider the one dimensional case in detail. They prove that, in their special case, the spectrum $\sigma\left(H_{\omega}\right)$ consists of the half line $[0, \infty)$ plus an additional isolated negative eigenvalue. This eigenvalue depends on the random parameters.

We will prove in the next section that in the higher dimensional case $(d>1)$ the spectrum is non-random under very mild assumptions. The main difference between $d=1$ and $d>1$ lies in the "ergodicity" of the potential under shifts parallel 
to the surface of the half space, which clearly does not apply to the one dimensional case.

Carmona [3] also considers the one dimensional case. He looks at the measure theoretic nature of the spectrum. Carmona proved the remarkable fact that, under suitable assumptions, $H_{\omega}=H_{0}+V_{\omega}$ has absolutely continuous resp. p.p. spectrum if $H_{\omega}^{+}$has ac. resp. p.p. spectrum near $E$ and $E \notin \sigma\left(H_{\omega}^{-}\right)$.

The only paper about the multidimensional case we are aware of is the paper [5] by Davies and Simon. While they treat only periodic $V_{\omega}^{ \pm}$this paper was one of our main motivations.

Our paper is organized as follows: In Sect. 2 we give some basic results about the spectrum of $H_{\omega}$. We prove that $\sigma\left(H_{\omega}\right)$ is non-random and contains the spectra $\sigma\left(H_{\omega}^{+}\right) \cup \sigma\left(H_{\omega}^{-}\right)$. In general, however, $\sigma\left(H_{\omega}\right)$ is bigger than $\sigma\left(H_{\omega}^{+}\right) \cup \sigma\left(H_{\omega}^{-}\right)$and we give a class of examples for this phenomenon. We call the energies in $\sigma\left(H_{\omega}^{+}\right) \cup \sigma\left(H_{\omega}^{-}\right)$ the bulk spectrum and the other energies in $\sigma\left(H_{\omega}\right)$ surface spectrum. This notation is justified by proving that points in the surface spectrum correspond to Weyl sequences concentrated near the surface $\left\{x_{1}=0\right\}$.

Section 3 discusses the density of states for $H_{\omega}$. We show that the integrated density of states for $H_{\omega}$ is nothing but the arithmetic mean of the density of states of $H_{\omega}^{+}$and $H_{\omega}^{-}$. Therefore the density of states is unable to detect the surface states. It is rather straightforward to conjecture that this is due to the fact that we normalize by a volume in the density of states while we should normalize by a surface term to grasp the surface states. This conjecture is proven in Sect. 3 and 4. In fact, we prove that there is a density of surface state which exists as a measure in the gaps of the bulk spectrum. Inside the bulk spectrum, the density of surface states exists in the sense of a next order correction to the (bulk) density of state. In this case, however, we can only prove existence in the sense of a (Schwarz) distribution.

The result about the density of surface states was already obtained for the Anderson model by two of the authors [7]. See also [8] and references therein for the consideration of special cases. Our results have been announced in [6].

In Sect. 5 we discuss some extensions and modifications of our results.

\section{Basic Definitions and Results}

Throughout this paper, we take $d \geqq 2$. Let $V_{\omega}(x), x \in \mathbb{R}^{d}$ be a random field on a probability space $(\Omega, \mathbb{F}, P) . V_{\omega}$ is called $\mathbb{R}^{d}$-stationary (respectively $\mathbb{Z}^{d}$-stationary) if there is a family $\left\{T_{i}\right\}_{i \in I}$ of measure-preserving transformations on $(\Omega, \mathscr{F}, P)$ with index set $I=\mathbb{R}^{d}$ (respectively $\mathbb{Z}^{d}$ ), such that $V_{T_{i} \omega}(x)=V_{\omega}(x-i)$. We call a random field stationary if it is $\mathbb{R}^{d}$-stationary or $\mathbb{Z}^{d}$-stationary. $V_{\omega}$ is called ergodic (respectively $\mathbb{R}^{d}$-ergodic, respectively $\mathbb{Z}^{d}$-ergodic) if the corresponding measure preserving transformations are ergodic, i.e. if any set $A \in \mathscr{F}$ invariant under all $T_{i}$ has probability zero or one. There is an easy procedure, the "suspension technique," to transfer results from the $\mathbb{R}^{d}$-ergodic case to the $\mathbb{Z}^{d}$-ergodic case almost automatically (see [9]). We will use suspension freely in what follows.

The general situation we consider in this paper is the following: $V_{\omega}^{+}$and $V_{\omega}^{-}$are two ergodic random fields on $\mathbb{R}^{d}$, independent of each other. We set for 
$x=\left(x_{1}, \ldots, x_{d}\right) \in \mathbb{R}^{d}:$

$$
V_{\omega}(x)=\left\{\begin{array}{lll}
V_{\omega}^{+}(x) & \text { for } & x_{1} \geqq 0 \\
V_{\omega}^{-}(x) & \text { for } & x_{1}<0
\end{array} .\right.
$$

$V_{\omega}$ is obviously not an ergodic potential. In fact, it is not even stationary. However, it is stationary with respect to those $T_{i}$ with $i_{1}=0$, i.e. for shifts perpendicular to the $x_{1}$-axis. While some of our results below remain true in a more general situation, we will suppose a further condition on the ergodic potentials $V_{\omega}^{ \pm}$which roughly speaking, ensures that no direction in space is distinguished by the process.

Definition. We call a family $\left\{T_{i}\right\}_{i \in I}\left(I=\mathbb{Z}^{d}\right.$ or $\left.\mathbb{R}^{d}\right)$ isotropically ergodic, if the families $T_{\Pi_{v} i}$ for $v=1, \ldots, d$ are ergodic, where $\Pi_{v}$ is the projection onto the $v^{\text {th }}$ coordinate axis.

Remark. It is easy to see that any mixing family is isotropically ergodic.

Examples. 1. Any periodic potential is an isotropic $\mathbb{Z}^{d}$-ergodic process (on a finite probability space).

2. Suppose that $g_{i}$ are i.i.d. random variables and that

$$
f \in l^{1}\left(L^{2}\right):=\left\{\varphi \mid \sum_{i \in \mathbb{Z}_{d}}\left(\int_{C_{0}}|\varphi(x-i)|^{2} d x\right)^{1 / 2}<\infty\right\}
$$

(where $C_{0}=\left\{x \in \mathbb{R}^{d} \mid-{ }^{1} / 2 \leqq x_{v} \leqq 1 / 2, v=1, \ldots, d\right\}$ ). Then the alloy-type potential

$$
V_{\omega}(x)=\sum g_{i}(\omega) f(x-i)
$$

is isotropically $\mathbb{Z}^{d}$-ergodic.

3. A homogeneous Gaussian process with correlation function vanishing at infinitely is isotropically ergodic.

Henceforth we assume $d \geqq 2$ and that $V^{ \pm}$are isotropically ergodic.

Theorem 1. The spectrum $\sigma\left(H_{\omega}\right)$ of $H_{\omega}$ is a non-random set (i.e. there is a set $\Sigma \subset \mathbb{R}$, s.t. $\Sigma=\sigma\left(H_{\omega}\right) P$-a.s. $)$. The same is true for the pure point, singular continuous, and absolutely continuous part of the spectrum. The discrete spectrum of $H_{\omega}$ is a.s. empty.

The proof is a not too difficult adjustment of the proof in [11] (see also Pastur [17] and Kunz-Souillard [14]). Another consequence of the ergodicity is the following result. Let us denote by $\Sigma^{ \pm}$the (a.s. constant) spectra of $H_{\omega}^{ \pm}$. Set $\Sigma_{0}=\Sigma^{+} \cup \Sigma^{-}$.

Theorem 2. The spectrum, $\Sigma$, of $H_{\omega}$ contains $\Sigma_{0}$.

Proof. Suppose $E \in \Sigma^{+}$. Then there is a Weyl sequence $\psi_{n},\left\|\psi_{n}\right\|=1$, $\left(\| H_{\omega}^{+}-E\right) \psi_{n} \| \rightarrow 0$ and $\psi_{n} \in \mathrm{C}_{0}^{\infty}\left(\mathbb{R}^{d}\right)$. Take $\varepsilon>0$ arbitrary. Denote by $K_{n}$ the compact support of $\psi_{n}$. Consider the set

$$
\Omega_{n, \varepsilon}=\left\{\omega \mid\left(\int_{K_{n}}\left|V_{\omega}(x)-V_{\omega}(x+(i, 0))\right|^{2} d x\right)^{1 / 2}<\varepsilon \text { for infinitely many } i \geqq 0\right\} .
$$

By Poincaré's recurrence theorem this event has probability one. Thus for $P$-almost all $\omega$ there exist $\tilde{\psi}_{n}(x)=\psi_{n}(x+i)$ such that $\operatorname{supp} \tilde{\psi}_{n} \subset\left\{x_{1}>0\right\},\left\|\tilde{\psi}_{n}\right\|=1$ and 
$\left(H_{\omega}^{+}-E\right) \tilde{\psi}_{n} \rightarrow 0$. Consequently $\tilde{\psi}_{n}$ is a Weyl sequence for $H_{\omega}$ and $E$, i.e. $E \in \sigma\left(H_{\omega}\right)$.

This result, of course, raises the question whether $\Sigma_{0}$ is already all of $\Sigma$. The question was considered in [5] for the special case of periodic $V_{\omega}^{ \pm}$. These authors found, in fact, additional spectrum in general. They called the corresponding states surface states, a notion we adopt. We call the corresponding energies in $\Sigma \backslash \Sigma_{0}$ the "surface energies," while we sometimes refer to $\Sigma_{0}$ as the "bulk spectrum."

To construct additional examples of $H_{\omega}$ with $\Sigma \backslash \Sigma_{0} \neq \phi$ we consider more closely the spectrum of $H_{\omega}$ in the case of alloy-type potentials, i.e.:

$$
V_{\omega}^{ \pm}(x)=\sum_{i \in \mathbb{Z}^{d}} q_{i}^{( \pm)}(\omega) f^{( \pm)}(x-i) .
$$

We assume that the $q_{i}^{+}, q_{j}^{-}$are independent with distributions $P_{0}^{ \pm}$and that the measures $P_{0}^{ \pm}$have compact support. Moreover, we assume that

$$
f^{( \pm)} \in l^{1}\left(L^{p}\right)=\left\{f \mid \sum_{i \in \mathbb{Z}^{d}}\left(\int_{C_{0}}|f(x-i)|^{p} d x\right)^{1 / p}<\infty\right\}
$$

with $C_{0}=\left\{x \mid 0 \leqq x_{i}<1 ; i=1, \ldots, d\right\}$ and some $p>\max \left(1, \frac{d}{2}\right)$. By $\mathscr{P}$ we denote the class of all potentials $W$ of the form:

$$
W_{\omega}(x)=\left\{\begin{array}{lll}
\Sigma \lambda_{i}^{-} f^{-}(x-i) & \text { for } & x_{1}<0 \\
\Sigma \lambda_{i}^{+} f^{+}(x-i) & \text { for } & x_{1} \geqq 0
\end{array},\right.
$$

with $\lambda_{i}^{ \pm}$periodic sequences (with some period) and $\lambda_{i}^{ \pm} \in \operatorname{supp} P_{0}^{ \pm}$. Following [7] or [12] it is not difficult to show:

$$
\text { Theorem 3. } \quad \sum=\bigcup_{W \in \mathscr{P}} \sigma\left(H_{0}+W\right) \text {. }
$$

Take now periodic potentials $V^{ \pm}=\Sigma \lambda^{ \pm} f^{ \pm}(x-i)$ and $V(x)=V^{ \pm}(x)$ for $\pm x_{1}>0$, such that $\sigma\left(H_{0}+V\right) \backslash\left(\sigma\left(H^{+}\right) \cup \sigma\left(H^{-}\right)\right) \neq \phi$. Potentials $V^{ \pm}$with this property can be constructed by the methods in [5]. Now we choose distribution $P_{0}^{ \pm}$concentrated close to $\lambda^{ \pm}$and consider $V_{\omega}(x)$ as in (2.2) with these distributions. Then, by the above theorem we have $\sigma\left(H_{0}+V\right) \subset \Sigma\left(=\sigma\left(H_{\omega}\right)\right)$. But by shrinking supp $P_{0}^{ \pm}$we can make $\sigma\left(H_{\omega}^{ \pm}\right)$arbitrarily close to $\sigma\left(H^{ \pm}\right)$. Thus by taking supp $P_{0}^{ \pm}$small enough we get $\Sigma \backslash\left(\sigma\left(H_{\omega}^{+}\right) \cup \sigma\left(H_{\omega}^{-}\right)\right) \neq \phi(-$ almost surely).

While we believe the notion of "surface energies" for points in $\Sigma \backslash \Sigma_{0}$ is rather intuitive, we will "justify" this notion further in various ways in the following. Recall that according to Weyl's criterion (see e.g. [18]) for any $E \in \sigma(H)$ there is a sequence $\psi_{n} \in L^{2}\left(\mathbb{R}^{d}\right)$ ("Weyl sequence") with $\left\|\psi_{n}\right\|=1$ and $H \psi_{n}-E \psi_{n} \rightarrow 0$. The next result tells us that a Weyl sequence for a surface energy remains close to the surface $\left\{x_{1}=0\right\}$. Here we can work in the following general setting: Suppose $V^{ \pm}$are operator bounded potential (with relative bounds less than 1). Set

$$
V(x)=\left\{\begin{array}{lll}
V^{-}(x) & \text { for } & x_{1}<0 \\
V^{+}(x) & \text { for } & x_{1} \geqq 0
\end{array}\right.
$$

and $\Sigma^{ \pm}=\sigma\left(H_{0}+V^{ \pm}\right), \Sigma_{0}=\Sigma^{+} \cup \Sigma^{-}, \Sigma=\sigma\left(H_{0}+V\right)$. Let us, furthermore, denote by $\chi_{R}$ the characteristic function of the set $S_{R}=\left\{x|| x_{1} \mid \leqq R\right\}$. 
Theorem 4. If $E \in \Sigma \backslash \Sigma_{0}$ and if $\psi_{n} \in C_{0}^{\infty}$ is a Weyl sequence for $H=H_{0}+V$ at energy $E$, then for any $R>0$,

$$
\lim _{n \rightarrow \infty}\left\|\chi_{R} \psi_{n}\right\|>0
$$

Proof. Suppose the assertion is wrong. By going over to a subsequence, if necessary, we may assume that

$$
\chi_{R} \psi_{n} \rightarrow 0
$$

It follows that also $\chi_{\frac{3}{4} R} \nabla \psi_{n} \rightarrow 0$. This can be seen as follows: Take $g \in C^{\infty}$, $0 \leqq g(x) \leqq 1$ such that $\Delta g$ is bounded and $g(x)=1$ for $\left|x_{1}\right| \leqq \frac{3}{4} R, g(x)=0$ for $\left|x_{1}\right| \geqq R$. Integrating by parts we get

$$
\begin{aligned}
\int g(x)\left|\nabla \psi_{n}\right|^{2} d x & \leqq \int|\Delta g(x)|\left|\psi_{n}\right|^{2} d x+\int g(x)\left|\psi_{n}\right|\left|\Delta \psi_{n}\right| d x \\
& \leqq M \int_{\left|x_{1}\right| \leqq R}\left|\psi_{n}(x)\right|^{2} d x+\left\|\Delta \psi_{n}\right\|\left(\int_{\left|x_{1}\right| \leqq R}\left|\psi_{n}(x)\right|^{2} d x\right)^{1 / 2}
\end{aligned}
$$

Choose now a $C^{\infty}$-function $\varrho, 0 \leqq \varrho \leqq 1$ with $\varrho(x)=1$ for $\left|x_{1}\right| \geqq \frac{3}{4} R$ and $\varrho(x)=0$ for $\left|x_{1}\right| \leqq \frac{R}{2}$, such that $\Delta \varrho$ and $\nabla \varrho$ are bounded. Then

$$
\left\|(H-E) \varrho \psi_{n}\right\| \leqq\left\|(H-E) \psi_{n}\right\|+2\left\|\nabla \varrho \nabla \psi_{n}\right\|+\left\|(\Delta \varrho) \psi_{n}\right\|
$$

Since both $\nabla \varrho$ and $\Delta \varrho$ have support in $S_{R}=\left\{x|| x_{1} \mid \leqq R\right\}$, the right side of (2.3) goes to zero. Consequently $\varrho \psi_{n}$ gives a Weyl sequence for $H^{+}$or $H^{-}$associated to $E$, hence $E \in \Sigma_{0}$ in contrast to our assumption.

Corollary. For any $\varepsilon>0$ there is an $R>0$ such that

$$
\lim \left\|\chi_{R} \psi_{n}\right\| \geqq 1-\varepsilon .
$$

Remark. Intuitively speaking, this corollary means that "surface states" are localized around the surface $x_{1}=0$.

Proof. Suppose the corollary is wrong for an $\varepsilon>0$, then (by going over to a subsequence) eventually

$$
\left\|\left(1-\chi_{n}\right) \psi_{n}\right\|>\varepsilon
$$

for all $n$. Let $g$ be a $C^{\infty}$-function on $\mathbb{R}$, such that $0 \leqq g(t) \leqq 1, g(1)=1$ for $t \geqq 1, g(t)=0$ for $t \leqq 1 / 2$ and set $\varrho(x)=g\left(x_{1}\right), \varrho_{n}(x)=\varrho\left(\frac{x}{n}\right)$. Then $\left\|\left(1-\varrho_{n}\right) \psi_{n}\right\| \geqq \varepsilon>0$ for all $n$ and

$$
\left\|(H-E)\left(1-\varrho_{n}\right) \psi_{n}\right\| \leqq\left\|\left(1-\varrho_{n}\right)(H-E) \psi_{n}\right\|+2\left\|\nabla \varrho_{n} \nabla \psi_{n}\right\|+\left\|\left(\Delta \varrho_{n}\right) \psi_{n}\right\|
$$

Since both $\nabla \varrho_{n}$ and $\Delta \varrho_{n}$ go to zero in sup-norm the right-hand side of (2.4) goes to zero, hence $\left(1-\varrho_{n}\right) \psi_{n}$ is a Weyl sequence.

\section{The Density of Surface States}

There are two equivalent ways to define the (integrated) density of states for an ergodic quantum mechanical disordered system. Let $H_{\omega}$ be a random Hamiltonian, with ergodic potential $V_{\omega \Lambda_{L}}$ a cube of side length $2 L$ centered at the origin. 
We denote by $\left(H_{\omega}\right)_{\Lambda_{L}}^{D}$ respectively $\left(H_{\omega}\right)_{\Lambda_{L}}^{N}$ the operator $H_{\omega}$ restricted to $L^{2}\left(\Lambda_{L}\right)$ with Dirichlet, respectively Neumann boundary conditions. It is easy to see that the functional

$$
f \mapsto \frac{1}{\left|\wedge_{L}\right|} \operatorname{tr} f\left(\left(H_{\omega}\right)_{\Lambda_{L}}^{D}\right) \quad \text { for } \quad f \in C_{0}(\mathbb{R})
$$

on the continuous functions of compact support defines a Borel measure $v_{L}^{D}$ on $\mathbb{R}$. Under mild assumptions on the potential $V_{\omega}$ it can be shown that $v_{L}^{D}$ converges vaguely to a measure $v$ as $L \rightarrow \infty$. Moreover, if we define $v_{L}^{N}$ in the same way replacing Dirichlet with Neumann boundary conditions, $v_{L}^{N}$ converges to the same limit. The measure $v$ is called the density of state measure for $H_{\omega}$.

The other method to define the density of states starts from the measures $\tilde{v}_{L}$ given by

$$
f \mapsto \frac{1}{\left|\Lambda_{L}\right|} \operatorname{tr}\left(\chi_{\Lambda_{L}} f\left(H_{\omega}\right)\right) .
$$

Again, it can be shown that $\tilde{v}_{L}$ converges vaguely as $L \rightarrow \infty$ and the limit is $v$. As one might expect from physical intuition the support, $\operatorname{supp} v$, of the density of states measure coincides with the spectrum $\Sigma\left(=\sigma\left(H_{\omega}\right)\right)$. For technical details we refer to $[16,1,2,13,4]$.

To be specific, we will assume throughout that for all $t>0$,

$$
\mathbb{E}\left(\int_{c_{0}} e^{-t V^{ \pm}(x)} d x\right)<\infty .
$$

This ensures the existence of the density of states measures $v^{ \pm}$of $H_{\omega}^{ \pm}$.

It is remarkably easy to prove the existence of the density of states $v$ also for the operator $H_{\omega}=H_{0}+V_{\omega}$, with $V_{\omega}$ given by (2.1) and to express it in terms of $v^{+}$and $v^{-}$.

Theorem 5. The density of state measure $v$ of $H_{\omega}=H_{0}+V_{\omega}$,

$$
V_{\omega}(x)=\left\{\begin{array}{lll}
V_{\omega}^{+}(x) & \text { for } & x_{1}<0 \\
V_{\omega}^{-}(x) & \text { for } & x_{1} \geqq 0
\end{array}\right.
$$

exists and is given by $v=\frac{1}{2} v^{+}+\frac{1}{2} v^{-}$.

Proof. Let us set $\Lambda_{L}^{+}=\left\{x \in \Lambda_{L} ; x_{1} \geqq 0\right\} \Lambda_{L}^{-}=\left\{x \in \Lambda_{L} ; x_{1}<0\right\}$. By DirichletNeumann bracketing (see $[20,13]$ ) we have

$$
\left(H_{\omega}\right)_{\Lambda_{L}}^{D} \leqq\left(H_{\omega}\right)_{\Lambda_{\bar{L}}}^{D}+\left(H_{\omega}\right)_{\Lambda_{L}^{+}}^{D}=\left(H_{\omega}^{-}\right)_{\Lambda_{\bar{L}}}^{D}+\left(H_{\omega}^{+}\right)_{\Lambda_{\bar{L}}^{+}}^{D}
$$

and

$$
\left(H_{\omega}\right)_{\Lambda_{L}}^{N} \geqq\left(H_{\omega}^{-}\right)_{\Lambda_{\bar{L}}}^{N}+\left(H_{\omega}^{+}\right)_{\Lambda_{L}^{+}}^{N} .
$$

Consequently, the distribution functions $N_{\Lambda_{L}}^{D}, N_{A_{L}}^{N}$ of $v_{L}^{D}$ and $v_{L}^{N}$ admit the estimate $(|A|$ denotes the Lebesgue measure of the set $A)$ :

$$
\begin{aligned}
\frac{1}{\left|\Lambda_{L}\right|} N_{\Lambda_{L}}^{D}(\lambda) & :=\frac{1}{L^{d}} \operatorname{tr} \chi_{(-\infty, \lambda)}\left(\left(H_{\omega}\right)_{\Lambda_{L}}^{D}\right) \\
& \leqq \frac{1}{2}\left\{\frac{1}{\left|\Lambda_{L}\right|} \operatorname{tr} \chi_{(-\infty, \lambda)}\left(\left(H_{\omega}^{-}\right)_{\Lambda_{\bar{L}}}^{D}\right)+\frac{1}{\left|\Lambda_{L}^{+}\right|} \operatorname{tr} \chi_{(-\infty, \lambda)}\left(\left(H_{\omega}^{+}\right)_{\Lambda_{L}^{+}}^{D}\right\}\right.
\end{aligned}
$$


and similarly

$$
\frac{1}{\left|\Lambda_{L}\right|} N_{\Lambda_{L}}^{N}(\lambda) \geqq \frac{1}{2}\left\{\frac{1}{\left|\Lambda_{L}\right|} \operatorname{tr} \chi_{(-\infty, \lambda)}\left(\left(H_{\omega}^{-}\right)_{\Lambda_{\bar{L}}}^{N}\right)+\frac{1}{\left|\Lambda_{L}^{+}\right|} \operatorname{tr} \chi_{(-\infty, \lambda)}\left(\left(H_{\omega}^{+}\right)_{\Lambda_{\bar{L}}}^{N}\right)\right\} .
$$

The right-hand side of (3.1) and (3.2) both converge to $\frac{1}{2}\left(\int_{-\infty}^{\lambda} d v_{L}^{+}+\int_{-\infty}^{\lambda} d v_{L}^{-}\right)$at all continuity points of the latter function; thus both $N_{\Lambda_{L}}^{N}(\lambda)$ and $N_{\Lambda_{L}}^{D}(\lambda)$ converge to this limit. It is well known that the vague convergence of the measure follows from the convergence of the distribution functions (at all continuity points of the limit).

The above result has as an immediate consequence that $\operatorname{supp}(v)=\Sigma_{0}$ which is (strictly) smaller than $\Sigma$ in general. This should not be too surprising from a physical point of view. It only tells us that the density of state is too rough a quantity to "see" the surface states. In fact, for an energy interval, $I$, to have nontrivial density of states measure, it is necessary that the number of states with energy in $I$ grows like the volume of the sample. For surface states it is however intuitively clear that their number should grow like a surface term.

Thus, instead of normalizing by the volume term $\left|\Lambda_{L}\right|=(2 L)^{d}$ we should rather normalize by a surface term $(2 L)^{d-1}$ which is just the area of the (hyper-)surface $\left\{x_{1}=0\right\}$ inside $\Lambda_{L}$. This is precisely how we define the density of surface states in $\Sigma \backslash \Sigma_{0}$. Obviously, for an interval $I \subset \Sigma_{0}$ the measures $\frac{\left|\Lambda_{L}\right|}{(2 L)^{d-1}} \tilde{v}_{L}$ cannot converge since $\tilde{v}_{L}$ converges to a nonzero limit. Therefore, inside the bulk spectrum, $\Sigma_{0}$, we define the density of surface states as the order $L^{d-1}$ correction to the bulk density of states (see below).

Definition. For a bounded function of compact support we set

$$
v_{L}^{S}(f):=\frac{1}{(2 L)^{d-1}} \operatorname{tr}\left\{\chi_{\Lambda_{L}^{+}}\left(f\left(H_{\omega}\right)-f\left(H_{\omega}^{+}\right)\right)+\chi_{\Lambda_{\bar{L}}}\left(f\left(H_{\omega}\right)-f\left(H_{\omega}^{-}\right)\right)\right\} .
$$

In other words $v_{L}^{S}$ just measures the deviation of $f\left(H_{\omega}\right)$ on $\Lambda_{L}$ from the direct sum of $f\left(H_{\omega}^{+}\right)$on $\Lambda_{L}^{+}$and $f\left(H_{\omega}^{-}\right)$on $\Lambda_{L}^{-}$.

We state our main result about the density of surface states:

Theorem 6. Suppose $f \in C^{3}(\mathbb{R})$ and $f(x)=0\left(e^{-a|x|}\right)$ for some $\alpha>0$. Then the limit

$$
v^{S}(f)=\lim _{L \rightarrow \infty} v_{L}^{S}(f)
$$

exists $P$-almost surely and is non-random. $v^{S}$ is a distribution of order (at most) 3.

The above defined $v^{s}$ is called the density of surface states (distribution). Before we begin the proof of Theorem 6 we turn to the behavior of $v^{s}$ in the "gaps" of $\Sigma^{0}$.

Corollary 7. $v^{s}$ restricted to $\mathbb{R} \backslash \Sigma_{0}$ is a positive measure which is finite on any compact subset of $\mathbb{R} \backslash \Sigma_{0}$. 
Remarks. 1. Because, as is intuitively clear, Dirichlet and Neumann boundary conditions introduce surface terms, we cannot use Dirichlet-Neumann breaketing to define a surface density of states.

2. Sometimes we will apply $v_{L}^{S}$ to functions of non-compact support, provided they decay sufficiently rapidly at infinity. We use this extension of the definition without further comment.

It is reasonable to call the limit of $v_{L}^{S}$ the density of surface states, provided this limit exists. In fact, below we will prove the existence of the limits $v_{L}^{S}$ (as $L \rightarrow \infty$ ) for functions $f$ that are sufficiently smooth. Therefore, we do not know whether the limit is a measure; we know, it is a distribution (of a certain order). We will however prove that it is a measure if restricted to the complement of $\Sigma_{0}$. We have no clear intuition whether this limitation is a drawback of our proof or whether $v^{S}$ is really not a measure. Let us, however, remark that $v^{S}$ certainly is not a positive measure, in general. In fact, it is not difficult to construct, in the spirit of Theorem 3, examples where $v^{S}(f)$ is negative for certain positive $f$. This is to be expected from physical reasoning and we might speak of "surface holes" in this case instead of surface states.

Proof (of the Corollary given the Theorem):

Take $f \in C^{3}, \operatorname{supp} f \subset \mathbb{R} \backslash \Sigma_{0}$ compact, $f \geqq 0$. Then

$$
\begin{aligned}
v_{L}^{S}(f) & =\frac{1}{(2 L)^{d-1}} \operatorname{tr}\left\{\chi_{\Lambda_{L}} f\left(H_{\omega}\right)-\chi_{\Lambda_{L}^{+}} f\left(H_{\omega}^{+}\right)-\chi_{\Lambda_{L}}-f\left(H_{\omega}^{-}\right)\right\} \\
& =\frac{1}{(2 L)^{d-1}} \operatorname{tr}\left\{\chi_{\Lambda_{L}} f\left(H_{\omega}\right)\right\} \geqq 0 .
\end{aligned}
$$

Observe that $f\left(H_{\omega}^{ \pm}\right)=0$ since $\sigma\left(H_{\omega}^{ \pm}\right) \cap \operatorname{supp} f=\phi$. Therefore the functional $v_{L}^{S}$ is positive and so is $v^{s}$. But, a positive functional on $C_{0}^{3}(\mathbb{R})$ is in fact (the integral) with respect to a positive measure by the Riesz representation theorem (and an inspection of its proof).

The rest of this section is devoted to the proof of Theorem 6 modulo an essentially deterministic result (Theorem 7 below) which is proven in the next section. To prove Theorem 6, we may restrict ourselves to the "right" part of $v_{L}^{S}(f)$, i.e. to prove the convergence of

$$
v_{L}^{S,+}:=\frac{1}{(2 L)^{d-1}} \operatorname{tr}\left\{\chi_{\Lambda_{L}^{+}}\left(f\left(H_{\omega}\right)-f\left(H_{\omega}^{+}\right)\right)\right\} .
$$

The other part can be handled in precisely the same way. Equation (3.3) can be written as a sum in the following way:

$$
\frac{1}{(2 L)^{d-1}} \sum_{j=0}^{L-1} \sum_{i \in \Lambda_{\underline{L}}^{1} \cap \mathbb{Z}^{d-1}} \operatorname{tr}\left\{\chi_{C_{(j, i)}}\left(f\left(H_{\omega}\right)-f\left(H_{\omega}^{+}\right)\right)\right\},
$$


where

$$
\Lambda_{L}^{1}=\left\{y \in \mathbb{R}^{d-1} \mid(x, y) \in \Lambda_{L} \text { for some } x \in \mathbb{R}\right\}
$$

(recall that $C_{(j, i)}=\left\{x \mid j \leqq x_{1} \leqq j+1, i_{v} \leqq x_{v}<i_{v}+1\right.$ for $\left.v=2, \ldots, d\right\}$ ). To shorten notation, we introduce

$$
\xi_{(j, i)}=\xi_{(j, i)}(f)=\operatorname{tr}\left\{\chi_{c_{(j, i)}}\left(f\left(H_{\omega}\right)-f\left(H_{\omega}^{+}\right)\right)\right\} .
$$

In the next section we will show that:

Theorem 7. $E\left(\left|\xi_{(j, i)}\right|\right) \leqq \frac{C}{1+j^{2}}$.

The constant $C$ depends on $f$.

Proof of Theorem 6. Given Theorem 7, we may write $v_{L}^{S,+}$ as:

$$
v_{L}^{S,+}=\frac{1}{L^{d-1}} \sum_{i \in \Lambda_{L}^{1} \cap \mathbb{Z}^{d-1}}\left(\sum_{j=0}^{\infty} \xi_{(j, i)}\right)-\frac{1}{L^{d-1}} \sum_{i \in \Lambda_{L}^{1} \cap \mathbb{Z}^{d-1}}\left(\sum_{j=L}^{\infty} \xi_{(j, i)}\right) \text {. }
$$

Let us set $\eta_{i}=\sum_{j=0}^{\infty} \xi_{(j, i)}$. By Theorem 7 the random variable $\eta_{i}$ exists and is integrable. Moreover $\eta_{i}$ is invariant under the shifts $T_{i}^{1}=T_{(0, i)}$. Consequently

$$
\frac{1}{L^{d-1}} \sum_{i \in \Lambda_{L}^{1} \cap \mathbb{Z}^{d-1}} \eta_{i}
$$

the first summand above, converges by Birkhoff's ergodic theorem. The second summand admits the estimate

$$
\mathbb{E}\left(\left|\frac{1}{L^{d-1}} \sum_{i \in \Lambda_{L}^{1} \cap \mathbb{Z}^{d-1}}\left(\sum_{j=L}^{\infty} \xi_{(j, i)}\right)\right|\right) \leqq \sum_{j=L}^{\infty} \mathbb{E}\left(\left|\xi_{j, i}\right|\right) \rightarrow 0 \text { as } L \rightarrow \infty .
$$

It follows from this and the Birkhoff theorem again that this term goes pointwise to zero $(P$-a.s.) as $L$ goes to infinity. This finishes the proof of Theorem 6 modulo Theorem 7.

\section{Proof of Theorem 7}

In this section we will prove Theorem 7 in a somewhat more general setting.

Suppose that $V^{ \pm}$are two potentials on $\mathbb{R}^{d}$ in the Kato class $K_{d}$ (see e.g. [21] or [4]). We set

$$
V(x)=\left\{\begin{array}{lll}
V^{-}(x) & \text { for } & x_{1}<0 \\
V^{+}(x) & \text { for } & x_{1} \geqq 0
\end{array}\right.
$$

and define $H^{ \pm}=H_{0}+V^{ \pm}$and $H=H_{0}+V$. As above we denote by $C_{(j, i)}$ for $j \in \mathbb{Z}$, $i \in \mathbb{Z}^{d-1}$ the cube

$$
C_{(j, i)}=\left\{x \mid 0 \leqq x_{1}-j<1,0 \leqq x_{v}-i_{v-1}<1, v=2, \ldots, d\right\} .
$$


We define $\xi_{(j, i)}(f)=\operatorname{tr}\left\{\chi_{c_{(j, i)}}\left(f(H)-f\left(H^{+}\right)\right\}\right.$for $j \geqq 0$ and with $H^{+}$replaced by $H^{-}$ for $j<0$.

Theorem $7^{\prime}$. For any $f \in C^{3}(\mathbb{R})$ with $f^{(l)}(x)=0\left(e^{-a x}\right)$ (with $a>0$ and $l=0,1,2,3$ ) as $x \rightarrow \infty$, there is a constant $C$ (depending only on $f$ and the $K_{d}$-norms of $V^{+}$) such that

$$
\left|\xi_{(j, i)}(f)\right| \leqq \frac{C}{1+j^{2}}
$$

We start the proof of Theorem $7^{\prime}$ by investigating the special case $f(x)=e^{-t x}$ for some $t>0$. Since $H^{ \pm}$and $H$ are bounded below we may assume that $H^{ \pm} \geqq 1$ and $H \geqq 1$ by adding a constant. In the rest of the proof we restrict ourselves to the case $j \geqq 0$, the other one being similar.

Proposition 1. For some $C, \alpha, \beta>0$,

$$
\left|\xi_{(j, i)}\left(e^{-t x}\right)\right| \leqq C e^{-\alpha j} e^{-\beta t}
$$

Proof. Set $\varphi(t):=\xi_{(j, i)}\left(e^{-t x}\right)$. Since $e^{-t H} \leqq e^{-t}$, we have for $t \geqq j$,

$$
|\varphi(t)|=\left|\operatorname{tr} \chi_{C(j, i)}\left(e^{-t H}-e^{-t H^{+}}\right)\right| \leqq 2 e^{-\frac{1}{2} t} e^{-\frac{1}{2} j}\left|\operatorname{tr}\left(\chi_{C(j, i)} e^{-\frac{t}{3} H}\right)\right| \leqq c e^{-\frac{1}{2} t} e^{-\frac{1}{2} j} .
$$

For $t<j$ we rely on a Feymann-Kac argument,

$$
\left.|\varphi(t)|=\mid \operatorname{tr} \chi_{C_{(j, i)}}\left(e^{-t H}-e^{-t H^{+}}\right)\right) \mid \leqq \int_{C_{(j, i)}} \mathbb{E}_{0,0}^{t, 0}\left\{\left|e^{-\int_{0}^{t} V(b(s)+x) d s}-e^{-\int_{0}^{t} V^{+}(b(s)+x) d s}\right|\right\} d x,
$$

where $\mathbb{E}_{0, x}^{t, y}$ denotes expectation over the Brownian bridge starting at time zero in $x$ and ending at time $t$ in $y$ (see [21] for more information). Unless the path reaches the negative half space the exponentials cancel so

$$
|\varphi(t)| \leqq \int_{C_{(j, i)}} \mathbb{E}_{0,0}^{1,0}\left\{\left(e^{-\int_{0}^{t} V(b(s)+x) d s}-e^{-\int_{0}^{t} V^{+(b(s)+x) d s}}\right) \chi\left\{b\left|\sup _{0 \leqq s \leqq t}\right| b_{1}(s) \mid>j\right\}\right\} d s .
$$

By the Schwarz inequality:

$$
\begin{gathered}
|\varphi(0)| \leqq 2 \int_{C_{(j, i)}} \mathbb{E}_{0,0}^{t, 0}\left\{e^{-\int_{0}^{t} V(b(s)+x) d s}+e^{-2 \int_{0}^{t} V^{+}(b(s)+x) d s}\right)^{1 / 2} d x \\
\mathbb{P}_{0,0}^{t, 0}\left(\sup _{0 \leqq s \leqq t}\left\{\left|b_{1}(s)\right|>j\right\}\right)^{1 / 2} .
\end{gathered}
$$

The first factor in the above formula is bounded since $V, V^{+} \in K_{d}$ by assumption. The second part can be estimated by:

$$
\mathbb{P}_{0,0}^{t, 0}\left(\sup _{0 \leqq s \leqq t}\left\{\left|b_{1}(s)\right|>j\right\}\right\} \leqq M e^{-\gamma \frac{j^{2}}{t}} \leqq M e^{-\gamma j} \leqq M e^{-\frac{\gamma}{2} j} e^{-\frac{\gamma}{2} t}
$$

The idea will be to analytically continue the estimates in $t$ and then use the Fourier transform. So, we consider the function

$$
\psi(z)=e^{\beta z} \xi_{(j, i)}\left(e^{-z \cdot}\right)
$$

as a function of the complex variable $z(\operatorname{Re} z \geqq 0)$. 


\section{Proposition 2.}

$$
|\psi(t+i s)| \leqq C e^{-\alpha j \arctan \frac{t}{|s|}}
$$

Proof. $\psi$ is analytic for $t=\operatorname{Re} z>0$ and $|\psi(z)| \leqq C_{2}$ for $\operatorname{Re} z \geqq 0$.

Moreover, from Proposition 1 we learn that

$$
|\psi(t+i s)| \leqq C e^{-\alpha j}
$$

From this we infer the assertion of the proposition by complex interpolation as follows: The transformation $\xi \mapsto e^{\xi}$ maps the strip $\{10 \leqq \operatorname{im} \xi \leqq \pi / 2\}$ into $\{z \mid \operatorname{Re} z \geqq 0, \operatorname{Im} z \geqq 0, z \neq 0\}$. By setting $\chi(\xi)=\psi\left(e^{\xi}\right)$. We define a function $\chi$ analytic in the above strip. We have

$$
\begin{gathered}
|\chi(\xi)| \leqq C_{1} e^{-\alpha j} \quad \text { for } \quad \operatorname{Im} v=0, \\
|\chi(\xi)| \leqq C_{2} \quad \text { for } \quad \operatorname{Im} \xi=\pi / 2 .
\end{gathered}
$$

Thus Hadamard's three line theorem (see e.g. [19]) implies that:

$$
|\chi(\xi)| \leqq C_{1}^{\operatorname{Im} \xi} C_{2}^{\pi / 2-\operatorname{Im} \xi} e^{-\alpha j(\pi / 2-\operatorname{Im} \xi)} .
$$

Since for $z=e^{\xi}$ we have that $\operatorname{Im} \xi=\arg z$, we get

$$
\begin{aligned}
|\psi(\xi)| & \leqq C_{1}^{\arg z} C_{2}^{\pi / 2-\arg z} e^{-\alpha j(\pi / 2-\arg z)} \\
& \leqq C e^{-\alpha j(\pi / 2-\arg z)}
\end{aligned}
$$

For $z=t+i s$ we have $\arg z=\arctan \frac{s}{t}$. Thus, we obtain the result for $s \geqq 0$. The argument for $s \leqq 0$ is the same.

The above result tells us that

$$
\left|\xi_{(j, i)}\left(e^{-(t+i s)}\right)\right| \leqq C e^{-\beta t} e^{-\alpha j \arctan \frac{t}{|s|}}
$$

We come to the proof for arbitrary $f \in C^{3}(\mathbb{R})$ with $f(x)=0\left(e^{-a x}\right)$ as $x \rightarrow \infty(a>0)$. Such a function can be written as

$$
f(x)=\frac{1}{x^{2}} g(x) \text { for } x>1 / 2 \text { (say), }
$$

where $g$ is of the same type. Let $g$ be the Fourier transition of $g$ normalized by

$$
g(x)=\int \tilde{g}(s) e^{-i s x} d s
$$

Then

$$
\begin{aligned}
f(H)-f\left(H^{+}\right) & =H^{-2} g(H)-\left(H^{+}\right)^{-2} g\left(H^{+}\right) \\
& =\int_{-\infty}^{+\infty}\left(H^{-2} e^{-i s H}-H_{+}^{-2} e^{-i s H^{+}}\right) \tilde{g}(s) d s \\
& =\int_{-\infty}^{+\infty} \tilde{g}(s) \int_{0}^{\infty} t\left(e^{-(t+i s) H}-e^{-(t+i s) H^{+}}\right) d t d s .
\end{aligned}
$$


Consequently

$$
\begin{aligned}
\left|\xi_{(j, k)}(f)\right| & =\left|\operatorname{tr}\left\{\chi_{(j, k)}\left(f(H)-f\left(H^{+}\right)\right)\right\}\right| \\
& =\mid \int_{-\infty}^{+\infty} \tilde{g}(s) \int_{0}^{\infty} t \operatorname{tr}\left\{\chi_{C_{(j, i)}}\left(e^{-(t+i s) H}-e^{-(t+i s) H^{+}}\right)\right\} d t d s \\
& \leqq \int_{-\infty}^{+\infty} \tilde{g}(s) \int_{0}^{\infty} t \mid \xi_{(j, i)}\left(\left(e^{-(t+i s) x}\right) \mid d t d s\right. \\
& \leqq C \int_{-\infty}^{+\infty} \tilde{g}(s) \int_{0}^{\infty} t e^{-\beta t} e^{-\alpha j \arctan \frac{t}{|s|}} d s d t .
\end{aligned}
$$

Using that

$$
\int_{0}^{\infty} t e^{-\beta t} e^{-\alpha j \arctan \frac{t}{|s|}} d t \leqq \frac{K\left(1+s^{2}\right)}{1+j^{2}}
$$

we conclude that

$$
(4.1) \leqq \frac{\widetilde{C}}{1+j^{2}} \int|\tilde{g}(s)|\left(1+s^{2}\right) d s \leqq \frac{\bar{C}}{1+j^{2}}
$$

for a $g$-dependent constant $\bar{C}$.

Note that the regularity assumption on $g$ is used to get $\int|\tilde{g}(s)|\left(1+s^{2}\right) d s$ finite, as well as to justify the Fourier inversion formula. (Recall what if $g, g^{\prime} \in L^{2}$ then $\tilde{g} \in L^{1}$.)

\section{Extensions and Modifications}

Let us consider once more the alloy type model with

$$
V_{\omega}^{ \pm}(x)=\sum q_{i}^{ \pm}(\omega) f^{ \pm}(x-i) \text {. }
$$

In the case the "mixed system" $V_{\omega}$ consisting of the system " - " in the left half space and of the system " + " in the right half space might be modelled by setting

$$
\widetilde{V}_{\omega}(x)=\sum_{i_{1}<0} q_{i}^{-}(\omega) f^{-}(x-i)+\sum_{i_{1} \geqq 0} q_{i}^{+}(\omega) f^{+}(x-i),
$$

which, of course, differs significantly from the $V_{\omega}$ discussed above.

Our results in the previous section still can be proven in this case by modifications of the proofs. To get the existence of the density of surface states, however, our proof seems to require $f$ to decay exponentially fast. While we feel this assumption is much stronger than necessary, we don't see how to avoid it. Similar considerations apply also for potentials with Poisson distributed sources.

It is not difficult to extend our theorem to cover discrete Schrödinger operators. In place of the path integral used in the continuum case, one can use a simple perturbation expansion in $H_{0}$.

Acknowledgements. H. Englisch gratefully acknowledges financial support through the Sonderforschungsbereich 237 "Unordnung und grosse Fluktuationen" and the Ruhr-Universität Bochum. He would like to thank especially S. Albeverio for his hospitality at the Ruhr-Universität 
Bochum. W. Kirsch thanks the Naturwissenschaftlich-Theoretisches Zentrum in Leipzig and the California Institute of Technology for financial support during visits at these institutions. Special thanks to G. Lassner, E. Stone, and D. Wales.

\section{References}

1. Avron, J., Simon, B.: Almost periodic Schrödinger operators. II. The integrated density of states. Duke Math. J. 50, 369-397 (1983)

2. Carmona, R.: Random Schrödinger operators. In: Lecture Notes in Mathematics, vol. 1180. Berlin Heidelberg New York: Springer 1986

3. Carmona, R.: One-dimensional Schrödinger operators with random or deterministic potentials: new spectral types. J. Funct. Anal. 51, 229 (1983)

4. Cycon, H., Froese, R., Kirsch, W., Simon, B.: Schrödinger operators. Berlin Heidelberg New York: Springer 1987

5. Davies, E.B., Simon, B.: Scattering theory for systems with different spatial asymptotics on the left and right. Commun. Math. Phys. 63, 277-301 (1978)

6. Englisch, H., Kirsch, W., Schröder, M., Simon, B.: Density of surface states in discrete models. Phys. Rev. Lett. 67, 1261-1262 (1988)

7. Englisch, J., Kürsten, K.D.: Infinite representability of Schrödinger operators with ergodic potentials. Anal. Auw. 3, 357-366 (1984)

8. Englisch, J., Schröder, M.: Bose condensation. II. Surface and bound states. Preprint

9. Kirsch, W.: On a class of random Schrödinger operators. Adv. Appl. Math. 6, 177-187 (1985)

10. Kirsch, W.: Random Schrödinger operators and the density of states. In: Lecture Notes in Mathematics, vol. 1109, Berlin Heidelberg New York: Springer 1985

11. Kirsch, W., Martinelli, F.: On the ergodic properties of the spectrum of general random operators. J. Reine Angew. Math. 334, 141-156 (1982)

12. Kirsch, W., Martinelli, F.: On the spectrum of Schrödinger operators with a random potential. Commun. Math. Phys. 85, 329 (1982)

13. Kirsch, W., Martinelli, F.: On the density of states of Schrödinger operators with a random potential. J. Phys. A 15, 2139-2156 (1982)

14. Kunz, H., Souillard, B.: Sur de spectre des operateurs aux differences finies aleatoires. Commun. Math. Phys. 78, 201-246 (1980)

15. Molcanov, S.A., Seidel, H.: Spectral properties of the general Sturm-Liouville equation with random coefficient. I. Math. Nacho. 109, 57-58 (1982)

16. Pastur, L.: Spectra of random selfadjoint operators. Russ. Math. Surv. 28, 1-67 (1973)

17. Pastur, L.: Spectral properties of disordered systems in one body approximation. Commun. Math. Phys. 75, 178 (1980)

18. Reed, M., Simon, B.: Methods of modern mathematical physics. I. Functional analysis (rev. ed.). New York: Academic Press 1980

19. Reed, M., Simon, B.: Methods of modern mathematical physics. II. Fourier analysis selfadjointness. New York: Academic Press 1975

20. Reed, M., Simon, B.: Methods of modern mathematical physics. IV. Analysis of operators. New York: Academic Press 1978

21. Simon, B.: Schrödinger semigroups. Bull Am. Math. Soc. 7, 447-526 (1982)

Communicated by T. Spencer

Received February 13, 1989 
